Original article

\title{
Y balance test has no correlation with the Stability Index of the Biodex Balance System
}

\author{
Gabriel Peixoto Leão Almeida*, Isabel Oliveira Monteiro, Débora Fortes Marizeiro, \\ Laísa Braga Maia, Pedro Olavo de Paula Lima \\ Federal University of Ceará, Department of Physical Therapy, Alexandre Baraúna Street, 949, Fortaleza, CE, Brazil
}

\section{A R T I C L E I N F O}

\section{Article history:}

Received 15 February 2016

Received in revised form

4 November 2016

Accepted 7 November 2016

\section{Keywords:}

Postural balance

Lower extremity

Evaluation

Biomechanics

\begin{abstract}
A B S T R A C T
Study design: A cross-sectional study design.

Background: The Stability Index of the Biodex Balance System (SI-BBS) and Y Balance Test (YBT) has been used in studies assessing postural stability but no studies have verified the association of the YBT with the SI-BBS.

Objective: To analyze the association of the Y Balance Test (YBT) with the Stability Index of the Biodex Balance System (SI-BBS) to evaluate postural stability.

Methods: Forty participants who engaged in recreational physical activities, 12 of whom had a history of injury to the lower limbs. Was used the SI-BBS and the anterior, posterolateral, posteromedial, and composite measures of the YBT. The order of execution of the tests and of the lower limbs evaluated was randomized and blind tested by two evaluators.

Results: Pearson's correlation coefficient was used to check the strength of the relationship between the distances achieved on the YBT and the SI-BBS. The YBT showed excellent reliability in the anterior, posteromedial, and posterolateral directions. However, the YBT showed no statistically significant correlation with any variables in the SI-BBS, indicating poor validity between YBT and SI-BBS assessments of postural stability in people with and without history of lower limb injuries.

Conclusions: The results of this study showed the YBT is not correlated with the SI-BBS as an assessment of postural stability. This finding has implications for researchers and clinicians using YBT results as the only measure of postural stability.
\end{abstract}

๑) 2016 Elsevier Ltd. All rights reserved.

\section{Introduction}

Postural stability can be defined as the ability to remain stable in static and dynamic body positions. Dynamic balance corresponds to maintaining stability while performing tasks and is essential for proper performance in daily living and sports activities (Bhat and Moiz, 2013; Greve et al., 2013). Postural stability is a complex process that depends on the interpretation of external stimuli received by sensory information mechanisms, including the visual, vestibular, and proprioceptive systems responsible for bringing

\footnotetext{
* Corresponding author. Alexandre Baraúna Street, 949, Rodolfo Teófilo, 60430160, Fortaleza, CE, Brazil.

E-mail addresses: gabriel_alm@hotmail.com (G.P.L. Almeida), isabelom55@ hotmail.com (I.O. Monteiro), debora.fortes@hotmail.com (D.F. Marizeiro), asial123@hotmail.com (L.B. Maia), pedrofisioterapia@hotmail.com (P.O. de Paula Lima).
}

information to the somatosensory cortex, where it is integrated to achieve neuromuscular control, maintenance of equilibrium, and suitable motor response (Kinzey and Armstrong, 1998; Hiemstra et al., 2001; Wassinger et al., 2014).

The Y Balance Test (YBT) is a functional assessment instrument for the lower limbs derived from the Star Excursion Balance Test. It is inexpensive and relatively quick and simple to execute. It is used to evaluate the dynamic stability of the lower limb, identify athletes at risk for injury, monitor the progress of rehabilitation, and perform neuromuscular training. It has been widely used to assess postural stability in adolescents (Holden et al., 2016), runners (Meardon et al., 2016) patients with ankle sprain (Ko et al., 2016), anterior cruciate ligament injury (Delahunt et al., 2013). The YBT requires lower limb strength, range of motion, and coordination and may be useful in predicting lesions in addition to assessing balance (Plisky et al., 2006, 2009; Filipa et al., 2010; Coughlan et al., 2012). 
The Biodex Balance System [Biodex, Inc., Shirley, NY] (BBS) consists of a mobile platform with 20 degrees of tilt in all directions and 12 levels of difficulty. It evaluates balance through a platform that oscillates in the anteroposterior, mediolateral, and overall directions simultaneously (Arnold and Schmitz, 1998; Son et al., 2013; Chen et al., 2014). Although the BBS is a tool that provides quick, objective test results, the equipment has a high cost, limiting its use in clinical practices where more accessible and lower cost evaluation instruments are available.

The Stability Index of the Biodex Balance System (SI-BBS) has been used in studies assessing postural stability (Arnold and Schmitz, 1998; Yamada et al., 2012), and it has shown moderate to high level of reliability in healthy subjects (Arifin et al., 2014), with patellofemoral pain (Akhbari et al., 2015), anterior cruciate ligament injury (Mohammadirad et al., 2012) and chronic low back pain (Sherafat et al., 2013). Y Balance Test has also shown good reliability levels for assessment of postural stability (Plisky et al., 2009). However, few studies have verified the correlation of stability measures of YBT with other assessment tools for the same outcome.

A recent study examined the correlation between the Star Excursion Balance Test and the Biodex Balance System Limits of Stability (Glave et al., 2016), but no studies have verified the association of the YBT with the SI-BBS. Therefore, the aim of this study was to analyze the association of the Y Balance Test with the Stability Index of the Biodex Balance System during the assessment of the postural stability. Our hypothesis was that the YBT would show moderate to good correlation with the SI-BBS.

\section{Materials and methods}

\subsection{Participants}

This study was a cross-sectional analysis of 40 individuals involved in physical activities. The participants were volunteers and consisted of 32 women ( $80 \%$ of the sample) and 8 men (20\% of the sample). All were between 18 and 30 years of age and were available for testing at the Human Movement Analysis Laboratory, Federal University of Ceará. Recreational physical activity was defined as any practiced sport in which a participant engaged for at least 30 min per day or for 150 min per week (Pate et al., 1995). This study was approved by the Ethics Committee at the Federal University of Ceará with protocol number 1.000.404. All of the participants provided written informed consent.

Individuals with and without a history of injury in the lower limbs were included. Candidates with disorders in the visual system or vestibular system, and those with neurological pathologies and/or orthopedic injuries that prevented testing were excluded. Our sample included 12 participants with injury history (age $=21.3$ years; weight $=55 \mathrm{~kg}$; height $=1.62 \mathrm{~m}$ ) and 28 participants without injury history (age $=20.8$ years; weight $=64.4 \mathrm{~kg}$; height $=1.64 \mathrm{~m}$ ) (Table 1$)$.

\subsection{Tests}

Participants were assessed using an evaluation form designed to gather data on anthropometric and clinical characteristics, along with information on their personal sports practices and history of injury. Those with a history of personal injury were also administered the Lower Extremity Functional Scale (LEFS) (Metsavaht et al., 2012).

Random Allocation Software (version 1.0.0) was used to randomize both the order in which the lower limbs were assessed (dominant or non-dominant) and the order in which the tests ( $Y$ Balance Test or Biodex Balance System) were administered. For comparison purposes, all assessments were conducted bilaterally. To standardize the measurement of lower extremity length, the distance between anterior superior iliac spine and the medial malleolus were used as reference points (Kinzey and Armstrong, 1998).

\subsection{1. $Y$ balance test}

Initial instructions for administering the YBT were provided to the evaluators. These verbal instructions provided information regarding the number of repetitions and positions participants should be asked to perform, along with directions and prohibitions for administering the assessment. The evaluator then showed the participant the test (Plisky et al., 2006, 2009).

The YBT assesses an individual's ability to maintain dynamic balance of the lower limb in the anterior, posteromedial, and posterolateral directions. All participants underwent the test following this sequence. To prevent fatigue, both limbs were tested in a single direction before introducing a new direction. Six trials were conducted as training, and the seventh was recognized as the test result (Plisky et al., 2009). During the six training repetitions, participant performance was informally evaluated, and participants were given feedback and guidance on test execution and avoiding mistakes. Between each practice set, participants were given $30 \mathrm{~s}$ of rest, and between changes in limb movement direction, 1 min of rest was given (Plisky et al., 2009; Hertel et al., 2010).

The test was carried out on a pad on which the volunteer was positioned. Strips of tape were fixed on the pad in the three directions to be evaluated. Each participant was asked to stand on one foot, with hands above hips, and to fix his or her gaze in the anterior

Table 1

Clinical and anthropometric characteristics of research participants (mean and standard deviation).

\begin{tabular}{|c|c|c|c|}
\hline & Total $(n=40)$ & Injury $(\mathrm{n}=12)$ & No injury $(\mathrm{n}=28)$ \\
\hline Age (years) & $20.9 \pm 2.5$ & $21.3 \pm 3.7$ & $20.8 \pm 2$ \\
\hline Weight (kg) & $62.1 \pm 11.1$ & $55.0 \pm 8.7$ & $64.4 \pm 10.9$ \\
\hline Height $(\mathrm{m})$ & $1.63 \pm 0.06$ & $1.62 \pm 0.04$ & $1.64 \pm 0.06$ \\
\hline $\operatorname{BMI}\left(\mathrm{kg} / \mathrm{m}^{2}\right)$ & $22.9 \pm 3.6$ & $20.9 \pm 3$ & $23.5 \pm 3.6$ \\
\hline Sports practice time (months) & $11.5 \pm 12.7$ & $16.4 \pm 16.7$ & $9.9 \pm 10.9$ \\
\hline Sports practice frequency (per week) & $4.1 \pm 1.1$ & $4.0 \pm 0.9$ & $4.1 \pm 1.1$ \\
\hline OSI (BBS) & $4.4^{\circ} \pm 1.9$ & $4.2^{\circ} \pm 1.5$ & $4.5^{\circ} \pm 2$ \\
\hline APSI (BBS) & $2.8^{\circ} \pm 1.6$ & $2.8^{\circ} \pm 1.8$ & $2.9^{\circ} \pm 1.6$ \\
\hline LMSI (BBS) & $2.9^{\circ} \pm 1.5$ & $2.6^{\circ} \pm 1.2$ & $3^{\circ} \pm 1.6$ \\
\hline Anterior (YBT) & $72.1 \pm 8.4 \mathrm{~cm}$ & $74.7 \pm 13.8 \mathrm{~cm}$ & $71.2 \pm 5.6 \mathrm{~cm}$ \\
\hline Posteromedial (YBT) & $71 \pm 13.5 \mathrm{~cm}$ & $74.2 \pm 21.9 \mathrm{~cm}$ & $69.9 \pm 9.2 \mathrm{~cm}$ \\
\hline Posterolateral (YBT) & $84.4 \pm 10.5 \mathrm{~cm}$ & $86 \pm 17.3 \mathrm{~cm}$ & $83.9 \pm 7 \mathrm{~cm}$ \\
\hline Composite (YBT) & $75.8 \pm 9.7 \mathrm{~cm}$ & $78.3 \pm 17.1 \mathrm{~cm}$ & $75 \pm 5.5 \mathrm{~cm}$ \\
\hline
\end{tabular}

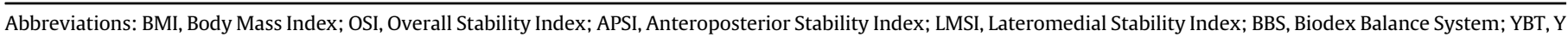
Balance Test. 


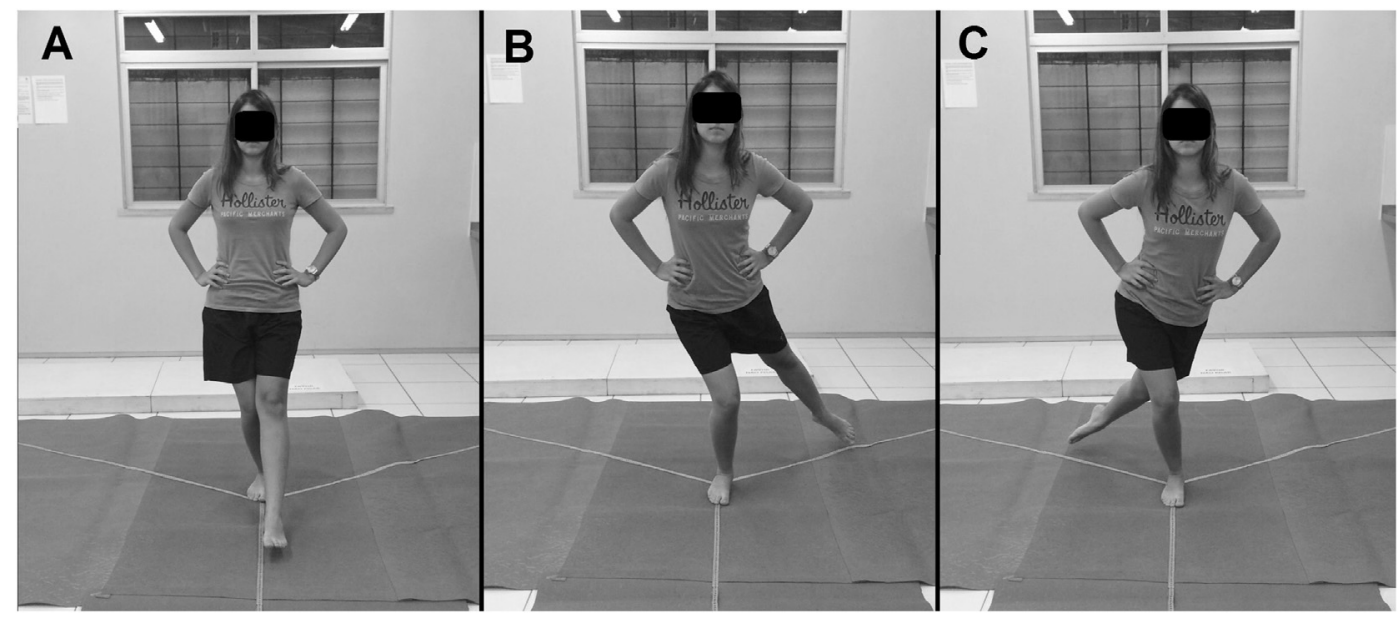

Fig. 1. Y Balance Test directions. A) Anterior; B) Posterolateral; C) Posteromedial.

direction. In the anterior direction, remained with the hallux at the point of intersection of the directions. During testing in the posteromedial and posterolateral directions, the participant was instructed to place his or her heel at the intersection of the three strips of tape (Fig. 1).

The participant was instructed to reach out as far as possible with his or her leg in the target direction without touching the tape or losing balance. Then returned to the resting position, then repeat the motion for continued practice. The seventh repetition was measured for distance.

A test result was considered invalid and discarded for any of the following reasons: failure to support the limb during the test, imbalance during the test, foot not completely in contact with the ground, foot of the reach leg contacting the ground, or imbalance during returning the reaching leg back to the starting position. If any of these failures occurred, the seventh (test) repetition was performed again (Plisky et al., 2006, 2009; Filipa et al., 2010).

In previous studies, the YBT intra-examiner reliability has been reported as moderate to good (ICC 0.67-0.97), and inter-examiner reliability as poor to good $(0.35-0.93)$ (Plisky et al., 2009). In this study, two assessors performed the YTB evaluations; interrater reliability was established with the intraclass correlation coefficient.

\subsubsection{Stability index of the Biodex Balance System}

The SI-BBS also was used to evaluate balance. The evaluation was performed with six different levels of platform stability, with $20 \mathrm{~s}$ in total. Level 6 was the most stable, and level 1 was the most unstable, consisting of $3.33 \mathrm{~s}$ at each level. The platform provides an objective assessment of balance using three indices: the overall stability index (OSI), an anteroposterior stability index (APSI), and a mediolateral stability index (MLSI). These indices are calculated according to the degree of platform oscillation; smaller values indicate the individual has good stability. The reported interexaminer reliability coefficients are 0.77 and 0.99 (Arnold and Schmitz, 1998; Hao and Chen, 2011; Yamada et al., 2012; Son et al., 2013; Chen et al., 2014).

The protocol was performed with participants in a unipodal stance. A training test was given to minimize the effects of learning, followed by three consecutive tests with $10 \mathrm{~s}$ between each (see Fig. 2). The mean of three tests was calculated and considered the result (Yamada et al., 2012).

Each participant was instructed regarding limb positioning, the number of repetitions required, and the form of test to be run, and

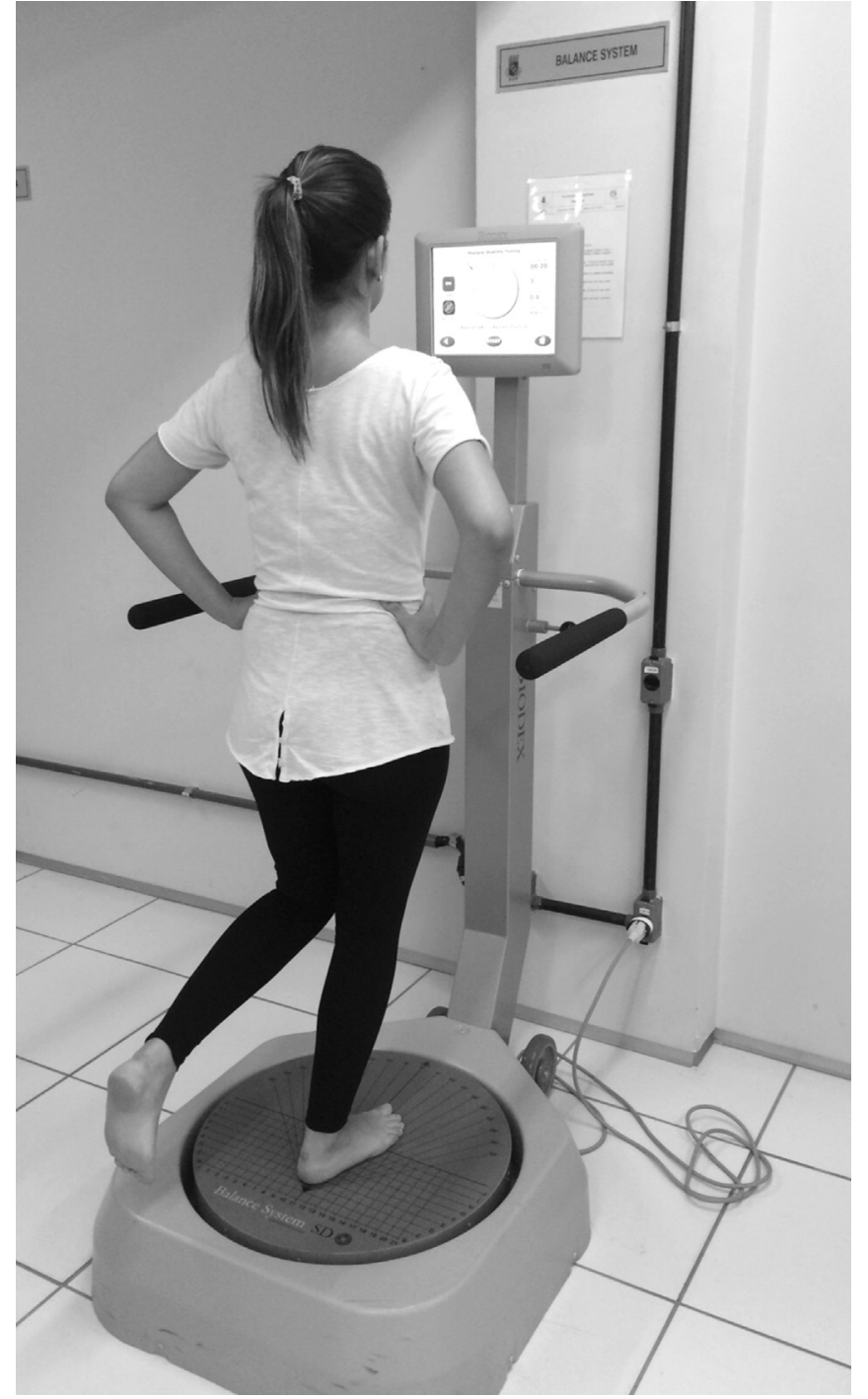

Fig. 2. Stability Index of the Biodex Balance System.

was encouraged to try to keep the platform in a neutral position. During the test, the participant was barefoot and remained on one 
foot, with hands parallel to the body and eyes open and fixed on the horizon (Cug and Wikstrom, 2014).

\subsection{Statistical analysis}

Data were analyzed using the program SPSS 17.0 (Statistical Package for the Social Sciences Inc., Chicago, IL, USA) with a significance value set at $5 \%$. The Kolmogorov-Smirnov test was used to verify the normality of the data distribution.

An independent $t$-test was used to assess whether there were differences between limb in participants with and without injury history as measured with the YBT, and a paired $t$-test was run to determine whether there were within-group differences among members. As neither test yielded significant differences, both limbs of all participants were used to assess the association of the YBT and the SI-BBS and to assess inter-examiner reliability on the YBT. Pearson's correlation coefficient to check the strength of the association between the distance achieved on the YBT and the SI-BBS, with coefficient values set as follows: $0-0.19=$ none to slight, $.2-0.39=$ low, $.4-0.69=$ modest, $.7-0.89=$ high, and $.9-1=$ very high (Weber and Lamb, 1970).

The inter-examiner reliability was assessed by the intraclass correlation coefficient. Reliability coefficients were interpreted as follows: $<0.69$ indicated poor interrater reliability, 0.70 to 0.79 signaled fair interrater reliability, 0.80 to 0.89 indicated good reliability, and 0.90 to 1.0 indicated excellent inter-examiner reliability (Cohen, 2013). We used three measures of agreement: the Bland and Altman plots, the Standard Error of the Measurement (SEM) and the Smallest Detectable Change (SDC). The SEM was calculated by dividing the standard deviation of the mean differences between the two measurements by the square root of 2 (SD differences $/ \sqrt{ } 2$ ), and the SDC was calculated using the formula SDC $=1.96 \times \sqrt{2} \times$ SEM. The SEM reflects the absolute error of the instrument, and the SDC reflects the smallest within-person change in a score that can be interpreted as a "real" change, above the measurement error of an individual (Terwee et al., 2007).

\section{Results}

The characteristics of the study participants are presented in Table 1. The intraclass correlation coefficient for the YBT showed excellent reliability in the anterior, posteromedial, and posterolateral directions (Table 2). The SEM and SDC are presented in Table 2.

The limits of agreement for the intra-examiner agreement ranged from $-4.5-3.1 \mathrm{~cm}$ (anterior); -5.5 to 3.9 (posteromedial); -6.5 to 2.2 (posterolateral); and -4.1 to 1.6 (composite), as shown by the Bland and Altman plots (Fig. 3).

The results of the measures of dynamic postural stability using the YBT showed none correlations with all balance variables on the SI-BBS, indicating poor association between the two measures for assessing balance in people with and without injury history in the lower limbs (Table 3).

Table 2

Inter-examiner reliability of Y Balance Test.

\begin{tabular}{lllll}
\hline & ICC & $95 \% \mathrm{CI}$ & SEM & SDC \\
\hline YBT anterior & 0.978 & $0.962-0.987$ & $0.25 \mathrm{~cm}$ & $1.38 \mathrm{~cm}$ \\
YBT posteromedial & 0.987 & $0.978-0.992$ & $0.31 \mathrm{~cm}$ & $1.54 \mathrm{~cm}$ \\
YBT posterolateral & 0.982 & $0.969-0.989$ & $0.29 \mathrm{~cm}$ & $1.49 \mathrm{~cm}$ \\
YBT composite & 0.991 & $0.985-0.995$ & - & - \\
\hline
\end{tabular}

Abbreviations: ICC: Intraclass Correlation Coefficient; CI: Confidente Interval; SEM: Standard Error of Measurement; SDC: Smallest Detectable Change.

\section{Discussion}

The aim of this study was to analyze the association of the $Y$ Balance Test with the Stability Index of the Biodex Balance System to determine whether they are equivalent measures useful for evaluating balance in people with and without a history of lower limb injury. Our study found none correlation between the YBT and the SI-BBS. This result limits the use of the YBT as the sole measure for evaluating balance.

The YBT is an instrument that is reported in the literature to be highly reliable, with reliability to the anterior 0.99; posteromedial 1.0; posterolateral 0.99; and; 0.97 composite (Plisky et al., 2009). The inter-examiner reliability obtained in this study was 0.97 for anterior; 0.98 for posteromedial; 0.98 for posterolateral, and 0.99 composite, corroborating the aforementioned study. Studies have used six replicates for training and one (Filipa et al., 2010) or three (Plisky et al., 2006) tests for evaluation. We chose to use six replicates of training and one test, in which we achieved excellent levels of reliability as reported in a previous study (Plisky et al., 2009). This high interrater reliability may be attributable to the practical training given the evaluators on the evaluation protocol.

The YBT is a balance assessment tool widely used in clinical practice and research mainly to track the progress of rehabilitation (Lee et al., 2014). A prospective study used the YBT to identify the risk of lower limb injury in 235 student basketball players. The results revealed that lower limb injury can be predicted with differences of $4 \mathrm{~cm}$ from normal values in the anterior, posteromedial, and posterolateral directions in women, and of $4 \mathrm{~cm}$ in the anterior direction in men (Plisky et al., 2006).

Other study evaluated the influence of neuromuscular training in YBT results. Women soccer players were assigned to a control group or to an experimental group given neuromuscular training. It was observed that the women given the neuromuscular training improved in YBT, confirming the test can be used to assess these variables (Filipa et al., 2010).

The hypothesis of the present study was that there would be moderate correlation between the YBT and the SI-BBS. However, none correlation evidenced by the statistical comparisons disconfirmed this hypothesis. This result may be due to different factors that influenced the assessment of balance in each test. In the case of the SI-BBS, an important factor in the assessment of balance oscillation is the pressure on the system in the anteroposterior and mediolateral directions simultaneously, which enables the equipment to check the amount of movement or center of gravity during the test. In the same way, in addition to measuring deviations in the center of pressure during static conditions, this device measures the degree of slope on each axle under dynamic conditions, thus providing more specific information of oscillation directions (Weber and Lamb, 1970; Yamada et al., 2012; Cug and Wikstrom, 2014). While YBT evaluates the participant's ability to achieve the maximum distance anteriorly, posterolateral and posteromedial in one leg support. Therefore, the YBT is a balance test more dynamic than the BBS. Moreover, YBT and the SI-BBS might measure different aspects of postural stability therefore limiting the congruence of the outcome. A recent study (Glave et al., 2016) examining the correlation between the Star Excursion Balance Test and the Biodex Balance System Limits of Stability Test returned negative correlations, indicating that participants who did well on one test did poorly on the other. It seems there is no "gold standard" for evaluating postural stability and the two tests used in this study can evaluate different magnitudes of postural stability, explaining the lack of association with each other.

Wassinger et al. (2014) evaluated the influence of immediate mobilization of the ankle on assessment results using the YBT. The authors suggested that manipulation of the foot or ankle can 


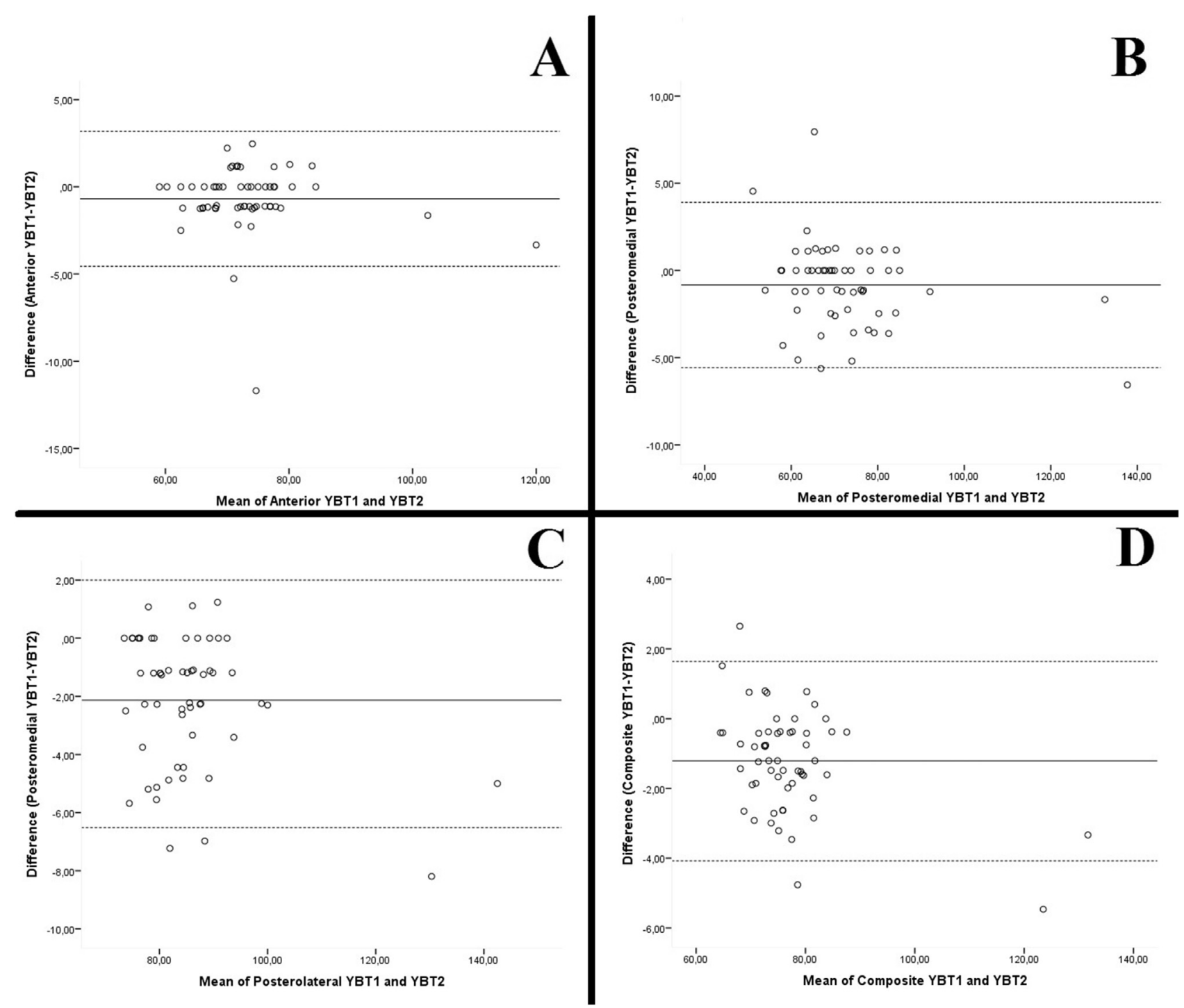

Fig. 3. Bland and Altman plots (YBT1 - YBT2) for (A) anterior; (B) posteromedial; (C) posterolateral; and (D) composite.

Table 3

Correlation between Y balance test and stability index of the Biodex Balance System.

\begin{tabular}{llll}
\hline & BBS APSI & BBS MLSI & BBS OSI \\
\hline YBT anterior & 0.105 & 0.005 & - \\
YBT posterolateral & -0.049 & 0.099 & - \\
YBT posteromedial & -0.049 & -0.001 & - \\
YBT composite & - & - & 0.016 \\
\hline
\end{tabular}

Abbreviations: BBS, Biodex Balance System; APSI, Anteroposterior Stability Index; LMSI, Lateromedial Stability Index; OSI, Overall Stability Index; YBT, Y Balance Test.

improve an individual's performance on the YBT. Given this finding, we infer that the range of motion of the ankle and foot may interfere with the results of the YBT. As range of motion is also intrinsically related to flexibility, lower limb flexibility is an additional variable that may affect the test's results.

In short, comparing the SI-BBS with the YBT clearly indicates both require coordination, proprioception, and balance, but the first is apparently more specifically designed to evaluate balance than the second.

A number of other variables, including foot type, ankle dorsiflexion range of motion, and quadriceps and gluteus medius strength can compromise the results and interpretation of the YBT as a solo tool for evaluation of balance (Gribble et al., 2012; Lee et al., 2014). Future studies should verify the relationship between the YBT and the SI-BBS and should include analysis of the confounding factors affecting YBT reliability.

Several factors limit the present study. First, our sample was predominantly composed of females (80\%), which may have affected the findings. In a retrospective study in which YBT results were obtained for 393 athletes from eight sports, it was observed that gender and sport can affect the result obtained (Stiffler et al., 2015). Another limitation was the implementation of the YBT. Since it is a dynamic test, an individual can compensate for balance and range limitations using other parts of the body, leading to inconsistent performance during the test and therefore less valid results for the individual. The test execution protocol used in this study was designed to minimize this effect; however, an assessment of the quality of movement during testing would help decrease the influence of such compensation.

\section{Conclusion}

The YBT has excellent inter-examiner reliability as a measure of balance in all directions. In this study, SEM and SDC were 
established for YBT; however, the YBT showed poor correlational validity with the SI-BBS. The results of this study showed the YBT is not correlated with the SI-BBS as an assessment of postural stability in participants with and without injury history. This finding has implications for researchers and clinicians using YBT results as the only measure of postural stability.

\section{References}

Akhbari, B., Salavati, M., Mohammadi, F., Safavi-Farokhi, Z., 2015. Intra- and intersession reliability of static and dynamic postural control in participants with and without patellofemoral pain syndrome. Physiother. Can. Physiother. Can. 67, 248-253.

Arifin, N., Abu Osman, N.A., Wan Abas, W.A., 2014. Intrarater test-retest reliability of static and dynamic stability indexes measurement using the Biodex Stability System during unilateral stance. J. Appl. biomechanics 30, 300-304.

Arnold, B.L., Schmitz, R.J., 1998. Examination of balance measures produced by the Biodex stability system. J. Athl. Train. 33, 323.

Bhat, R., Moiz, J.A., 2013. Comparison of dynamic balance in collegiate field hockey and football players using star excursion balance test. Asian J. sports Med. 4, 221.

Chen, T.-H., Chou, L.-W., Tsai, M.-W., Lo, M.-J., Kao, M.-J., 2014. Effectiveness of a heel cup with an arch support insole on the standing balance of the elderly. Clin. interventions aging 9, 351 .

Cohen, J., 2013. Statistical Power Analysis for the Behavioral Sciences. Academic press.

Coughlan, G.F., Fullam, K., Delahunt, E., Gissane, C., Caulfield, B.M., 2012. A comparison between performance on selected directions of the star excursion balance test and the Y Balance test. J. Athl. Train. 47, 366.

Cug, M., Wikstrom, E.A., 2014. Learning effects associated with the least stable level of the Biodex ${ }^{\mathbb{R}}$ stability system during dual and single limb stance. J. sports Sci. Med. 13, 387.

Delahunt, E., Chawke, M., Kelleher, J., Murphy, K., Prendiville, A., Sweeny, L., et al., 2013. Lower limb kinematics and dynamic postural stability in anterior cruciate ligament-reconstructed female athletes. J. Athl. Train. 48, 172-185.

Filipa, A., Byrnes, R., Paterno, M.V., Myer, G.D., Hewett, T.E., 2010. Neuromuscular training improves performance on the star excursion balance test in young female athletes. J. Orthop. sports Phys. Ther. 40, 551-558.

Glave, A.P., Didier, J.J., Weatherwax, J., Browning, S.J., Fiaud, V., 2016. Testing postural stability: are the star excursion balance test and Biodex balance system limits of stability tests consistent? Gait posture 43, 225-227.

Greve, J.M.D.A., Cuğ, M., Dülgeroğlu, D., Brech, G.C., Alonso, A.C., 2013. Relationship between anthropometric factors, gender, and balance under unstable conditions in young adults. BioMed Res. Int. 2013.

Gribble, P.A., Hertel, J., Plisky, P., 2012. Using the Star Excursion Balance Test to assess dynamic postural-control deficits and outcomes in lower extremity injury: a literature and systematic review. J. Athl. Train. 47, 339-357.

Hao, W.-Y., Chen, Y., 2011. Backward walking training improves balance in schoolaged boys. BMC Sports Science. Med. Rehabilitation 3, 24.

Hertel, J., Miller, S.J., Denegar, C.R., 2010. Intratester and Intertester Reliability during the Star Excursion Balance Tests. JSR, p. 9.
Hiemstra, L.A., Lo, I.K., Fowler, P.J., 2001. Effect of fatigue on knee proprioception: implications for dynamic stabilization. J. Orthop. Sports Phys. Ther. 31 598-605.

Holden, S., Boreham, C., Doherty, C., Wang, D., Delahunt, E., 2016. A longitudina investigation into the progression of dynamic postural stability performance in adolescents. Gait posture 48, 171-176.

Kinzey, S.J., Armstrong, C.W., 1998. The reliability of the star-excursion test in assessing dynamic balance. J. Orthop. Sports Phys. Ther. 27, 356-360.

Ko, J., Rosen, A.B., Brown, C.N., 2016. Comparison between single and combined clinical postural stability tests in individuals with and without chronic ankle instability. Clin. J. sport Med. official J. Can. Acad. Sport Med.

Lee, D.-K., Kim, G.-M., Ha, S.-M., Oh, J.-S., 2014. Correlation of the Y-Balance test with lower-limb strength of adult women. J. Phys. Ther. Sci. 26, 641-643.

Meardon, S., Klusendorf, A., Kernozek, T., 2016. Influence of injury on dynamic postural control in runners. Int. J. sports Phys. Ther. 11, 366-377.

Metsavaht, L., Leporace, G., Riberto, M., Sposito, M.M.M., Del Castillo, L.N. Oliveira, L.P., et al., 2012. Translation and cross-cultural adaptation of the lower extremity functional scale into a Brazilian Portuguese version and validation on patients with knee injuries. J. Orthop. Sports Phys. Ther. 42, 932-939.

Mohammadirad, S. Salavati, M., Takamjani, I.E., Akhbari, B., Sherafat, S. Mazaheri, M., et al., 2012. Intra and intersession reliability of a postural contro protocol in athletes with and without anterior cruciate ligament reconstruction: a dual-task paradigm. Int. J. sports Phys. Ther. 7, 627-636.

Pate, R.R., Pratt, M., Blair, S.N., Haskell, W.L., Macera, C.A., Bouchard, C., et al., 1995 Physical activity and public health: a recommendation from the centers for disease control and prevention and the american college of sports medicine. Jama 273, 402-407.

Plisky, P.J., Gorman, P.P., Butler, R.J., Kiesel, K.B., Underwood, F.B., Elkins, B., 2009. The reliability of an instrumented device for measuring components of the star excursion balance test. North Am. J. sports Phys. Ther. NAJSPT 4, 92.

Plisky, P.J., Rauh, M.J., Kaminski, T.W., Underwood, F.B., 2006. Star Excursion Balance Test as a predictor of lower extremity injury in high school basketball players. J. Orthop. Sports Phys. Ther. 36, 911-919.

Sherafat, S., Salavati, M., Ebrahimi Takamjani, I., Akhbari, B. Mohammadirad, S., Mazaheri, M., et al., 2013. Intrasession and intersession reliability of postural control in participants with and without nonspecific low back pain using the Biodex Balance System. J. Manip. physiological Ther. 36, 111-118.

Son, S.M., Kang, K.W., Lee, N.K., Nam, S.H., Kwon, J.W., Kim, K., 2013. Influence of isokinetic strength training of unilateral ankle on ipsilateral one-legged standing balance of adults. J. Phys. Ther. Sci. 25, 1313.

Stiffler, M.R., Sanfilippo, J.L., Brooks, M.A., Heiderscheit, B.C. 2015. Star excursion balance test performance varies by sport in healthy division I collegiate athletes. J. Orthop. Sports Phys. Ther. 1-30.

Terwee, C.B., Bot, S.D., de Boer, M.R., van der Windt, D.A., Knol, D.L., Dekker, J., et al, 2007. Quality criteria were proposed for measurement properties of health status questionnaires. J. Clin. Epidemiol. 60, 34-42.

Wassinger, C.A., Rockett, A., Pitman, L., Murphy, M.M., Peters, C., 2014. Acute effects of rearfoot manipulation on dynamic standing balance in healthy individuals. Man. Ther. 19, 242-245.

Weber, J.C., Lamb, D.R., 1970. Statistics and Research in Physical Education. CV Mosby Company.

Yamada, R.K.F., Arliani, G.G., Almeida, G.P.L., Venturine, A.M., CVd, Santos, Astur, D.C., et al., 2012. The effects of one-half of a soccer match on the postura stability and functional capacity of the lower limbs in young soccer players. Clinics 67, 1361-1364. 